\title{
Physical load affects duration judgments: A meta-analytic review
}

\author{
Authors: Richard A. Block, P.A. Hancock, \& Dan \\ Zakay
}

NOTICE: this is the author's version of a work that was accepted for publication in Acta Psychologica. Changes resulting from the publishing process, such as peer review, editing, corrections, structural formatting, and other quality control mechanisms may not be reflected in this document. Changes may have been made to this work since it was submitted for publication. A definitive version was subsequently published in Acta Psychologica, [VOL\# 165, (Marcg 2016)] DOI\# 10.1016/j.actpsy.2016.01.002

Block, Richard A. , P.A. Hancock, and Dan Zakay. "Physical load affects duration judgments: A meta-analytic review." Acta Psychologica 165 (March 2016): 43-47. DOI: 10.1016/j.actpsy.2016.01.002.

Made available through Montana State University's ScholarWorks scholarworks. montana.edu 


\title{
Physical load affects duration judgments: A meta-analytic review
}

\author{
Richard A. Block ${ }^{\mathrm{a}}$, P.A. Hancock ${ }^{\mathrm{b}}$, \& Dan Zakay \\ ${ }^{a}$ Montana State University, Bozeman, MT 59717-3440, United States \\ b University of Central Florida, Orlando, FL 32816-1390, United States \\ ' Interdisciplinary Center Herzliya, Herzliya, Israel
}

\begin{abstract}
This article reports a meta-analytic review of seven extant experiments, with 235 participants, concerning effects of physical workload on duration judgments. It also provides a qualitative assessment of related studies that, for specific reasons, were not includable in the quantitative meta-analysis. All analyzed experiments used the prospective duration-judgment paradigm and the production method, in which participants knew in advance that duration estimation was required. A large overall effect size reveals that increasing physical workload results in longer prospective duration productions. Physical workload effects are comparable to those of cognitive load. Implications for applied research, theory, and applications are discussed.
\end{abstract}

\section{Introduction}

People encounter combinations of physical and cognitive workload in many aspects of life. People attempting to cross a street while carrying a heavy basket from a supermarket must estimate their time for passage to safety. Basketball players must scan for other players while concurrently estimating the duration during which they are allowed to remain in the restricted area of the lane, as well as the maximum duration allowed to make a shot. People operating complex technologies, such as airplane pilots and automobile drivers, also encounter increasing levels of both physical and cognitive workload as they navigate crowded airways and highways. They must do this in spite of new computer-assisted interfaces that mediate multiple tasks which often demand divided attention. Computer-control systems, such as automatic-pilots, GPS devices, and other in-vehicle assistive systems can be useful. However, they often compete for limited attentionand time-constrained resources with ongoing psychomotor control demands. Firefighters and military personnel often must carry heavy physical loads while they are concurrently making operational decisions. Many people time-share and attention-share other tasks, such as using their personal electronic devices, as they are driving or walking. Increasingly, people must perform concurrent physical and cognitive tasks, and this trend will probably continue and increase.
Most researchers agree that both cognitive and physical load consume attentional resources. In both cases, concurrent duration judgment should be reduced more when workload is high than when it is low. This is explained by attentional models of prospective duration judgment (e.g., Zakay \& Block, 1997), according to which duration judgment is a function of the overall number of pulses which are emitted by a pacemaker-clock and accumulated in a counter. The number of pulses that pass through to the counter in a time unit is controlled by the amount of attentional resources allocated for timing. Attentional resources, however, are competing with other concurrent tasks since they are all coming from a general pool. When cognitive or physical load is high, less attentional resources are left for timing and vice versa. This was validated regarding cognitive load (e.g., Block, Hancock, \& Zakay, 2010). However, after more than a century of experimental investigations (Hancock \& Block, 2012), there is surprisingly little systematic evaluation of physical load effects.

Our recent meta-analytic review reveals that cognitive load influences duration judgments in a systematic and reliable way (Block et al., 2010). Duration estimation is therefore a useful and sensitive index of information-processing load (Zakay, Block, \& Tsal, 1999). In the present meta-analytic review, we report the effects of physical load on duration judgments and compare such effects to our previously reported pattern for that on cognitive load.

All usable studies included and reviewed in the present metaanalysis used the prospective duration-judgment paradigm, in which participants are aware that they will be required to estimate a time period of various tasks (Zakay \& Block, 1997). This paradigm is very 
similar to procedures to estimate time-to-contact (Hancock \& Manser, 1997). However, production stands in contrast to the retrospective paradigm, in which participants are initially unaware that duration estimates will subsequently be required. All reviewed experiments used a design in which high physical load and low physical load tasks were performed in either a randomized or a counterbalanced order. All used the method of production, in which participants are asked to make a response at when the experimenter-defined duration has ended. This method is sensitive and informative (Hancock, Vercruyssen, \& Rodenburg, 1992; Zakay et al., 1999). It stands in contrast to verbal estimation, in which participants are asked to estimate an elapsed duration in standard time units, such as minutes and seconds. The methods of production and verbal estimation are thought to be inversely related (Horenstein \& Rotter, 1969).

To manipulate physical load, the reviewed experimenters have used a wide variety of tasks. For example, Weybrew (1963) and Warm, Smith, and Caldwell (1967) used a dynamometer, with some participants told to exert near-maximum force (high load) and other participants told to exert minimal force (low load). Comparisons were made across these respective load levels. Other researchers compared performance in an airplane flight simulator, using either a difficult flight plan or an easy flight plan (e.g., Bortolussi, Kantowitz, \& Hart, 1986). These researchers examined performance using either difficult or easy simulator-based tasks to investigate psychomotor demands. Still other researchers (e.g., Arlin, 1989) studied children estimating durations while carrying heavy or light pipes.

There is little systematic research that could be included in the quantitative meta-analysis. Considering the necessities of demands such as multitasking, this is surprising. Our present meta-analysis not only summarizes what is presently known, but it also serves as a benchmark, indicating what remains to be discovered. Consequently, here we do not simply summarize extant information; our work looks to inform experimenters how much and what remains to be done in this area of growing theoretical and applied importance. Our hypothesis is that the impact of physical load on prospective duration judgment will be found to be similar to that of cognitive workload, namely, that the higher physical load is, the shorter prospective duration productions will be.

\section{Method}

\subsection{Selection of experiments}

We used the same selection strategy and meta-analytic methods as in our previous meta-analytic research on effects of cognitive load (Block et al., 2010). We searched more than 14,000 references on the psychology of time, including references from two major databases, PsycINFO (1887-2013), using the keywords time perception and time estimation, and Medline (1966-2013), using the keyword time perception. We also searched published bibliographies on time research, book chapters, and books; as well as each of our individual files. We searched for articles that contained such terms as duration judgment, workload, physical load, cognitive load, attention, difficulty, as well as many other similar and related terms.

We checked the reference lists of relevant articles to ascertain whether other studies might also be included. We included only experiments that manipulated physical load from which we were able to calculate an effect size from reported inferential statistics, a data table, or a figure. Our inclusion and exclusion criteria were identical to those used in our previous meta-analyses of duration judgments (e.g., Block et al., 2010). Every included experiment involved normal human participants judging durations predominantly equal to or greater than $3 \mathrm{~s}$, with at least one of the independent variables involving physical load as defined earlier. Perception and estimation of durations less than about $3 \mathrm{~s}$ involve very different processes than of longer durations (for reviews and evidence, see Hancock, Arthur, Chrysler, \& Lee, 1994; Wittmann, 1999), and thus our exclusion threshold at this value.

\subsection{Effect size analyses}

We independently identified effect sizes, and we resolved any disagreements by discussion. Each effect size was calculated as $g$, the sample-size-corrected difference between the mean duration judgments given by participants in each condition divided by the pooled standard deviation (Hedges, 1981; Hedges \& Olkin, 1985), using Borenstein, Hedges, Higgins, and Rothstein's (2011, Version 2.2.064) Comprehensive Meta-Analysis (CMA) program. Effect sizes were defined as positive if the duration judgment ratio was larger under the high physical-load condition as compared with that under the low physical-load condition. Conversely, it was defined as negative if the duration judgment ratio was smaller under high rather than low physical load conditions. This was the case in all experiments we analyzed (see Block et al., 2010, for details, including on what negative effect sizes mean). Negative effect sizes reveal that time productions were shorter under high-workload than under low-workload conditions.

The meta-analytic procedures and calculations were the same as in Block et al. (2010). However, because of the small number of studies, we could not analyze any moderator-variable effects. Given such a limitation, we also review other relevant and important studies in a qualitative way.

\section{Results}

Seven includable experiments, with a total of 235 participants, manipulated physical load. All used the prospective duration-judgment paradigm and the method of production.

As shown in Table 1 and Fig. 1, each of the studies show a negative effect size, and each experiment revealed a significant (or marginally significant) effect. Many effect sizes are large, whereas others are medium (Cohen, 1988). Confidence intervals reveal the consistency across this data set. Considering the limited number of experiments for which an effect size could be calculated, a qualitative assessment follows later in the General discussion section.

Using a random-effect model, as is recommended, the overall weighted mean effect size was significant $\left(d_{+}=-1.36,95 \%\right.$ $\mathrm{CI}=-2.06$ to $-0.65, p<.001)$. This overall effect is larger than the comparable overall effect for cognitive load reported by Block et al. (2010). Because the cumulative confidence interval does not include zero (see Fig. 1), substantive and consistent effects of interest need to be explained.

\section{General discussion}

The present meta-analytic study concerned ongoing theoretical controversies about duration judgment processes by investigating possible effects of physical workload on prospective time estimates. In particular, we investigated whether the effect of physical workload on duration judgment is similar to the effect of cognitive load by using exactly the same meta-analytic procedures as in our previous research (Block et al., 2010). Our meta-analysis showed an overall effect size $\left(d_{+}=-1.36\right)$ that is comparable to and in the same direction as the effects of cognitive load on prospective duration. This reveals that high physical load demands require attention, which lengthens prospective timing (i.e., shortens time productions). It may be attributable to the fact that tasks involving high physical load, such as piloting an aircraft, demands the allocation of attention at the same time that they involve the sequencing and executing of complex physical movements. Therefore, when relatively high amounts of physical efforts and cognitive functions are combined in a dual-task situation, there are relatively fewer residual attentional resources available to allocate to timing. If both cognitive and physical loads are combined (e.g., in a car or an airplane simulator), the effect on time production is greater. This implies that even though the processes behind the two types of load are not entirely the same, the effects are interactive. This hypothesis is supported 
Table 1

Summary of experiments in the present meta-analysis.

\begin{tabular}{|c|c|c|c|c|c|}
\hline Author(s) and date & $g$ & $95 \% \mathrm{CI}$ & $p$ & $\mathrm{~N}$ & Method (abbreviated) \\
\hline Weybrew (1963) & -0.65 & -1.29 to -0.02 & .039 & 20 & Gripping dynamometer \\
\hline Warm et al. (1967) & -1.02 & -1.68 to -0.36 & .002 & 20 & Gripping dynamometer \\
\hline Bortolussi et al. (1986) & -2.70 & -3.81 to -1.60 & $<.001$ & $\ldots 12$ & Piloting in airplane simulator \\
\hline Arlin (1989) & -0.34 & -0.58 to -0.11 & .005 & 140 & Transferring heavy/light pipes \\
\hline Vercruyssen, Hancock, and Mihaly (1989) & -0.71 & -1.57 to +0.15 & .091 & 11 & Exercising on cycle ergometer \\
\hline Baldauf, Burgard, and Wittmann (2009) & -2.24 & -2.99 to -1.49 & $<.001$ & 16 & Driving in car simulator \\
\hline Buscà, Moras, Seirulo-Lo, and Cabot (2011) & -2.21 & -2.96 to -1.47 & $<.001$ & 16 & Walking under different loads \\
\hline Overall (fixed model) & -0.77 & -0.95 to -0.58 & $<.001$ & 235 & \\
\hline Overall (random model) & -1.36 & -2.06 to -0.65 & $<.001$ & 235 & \\
\hline
\end{tabular}

Note. All effect sizes ( $g$ values) were negative, which reveals that time productions were longer under high physical load than under low physical load.

by a study (Paas \& Adam, 1991) in which participants performed both a decision-making task and a perceptual task. These tasks were performed either with or without accompanying physical exercise. The findings revealed that the performance on the perceptual task was impaired by physical load, but the performance on the decision-making task was improved in the presence of physical load demands. This can be explained by the nature of the resources required by physical exercises, which competes to influence perception but not to influence higher cognitive tasks like decision-making. Prospective timing is similar to perceptual tasks (Zakay, Bibi, \& Algom, 2014) in many ways and thus it is reasonable to assume that timing should also be affected by concurrent physical load. This effect is similar to the effect of pain, which has also been found to interrupt and demand attention (Eccleston \& Crombez, 1999). It suggests that there is a need to carefully parse the effects of physical load from its cognitive component, as well as vice-versa.

In the present meta-analytic review, we investigated effects of physical workload on prospective duration judgments, all of which used the method of production. Several methods are available for measuring workload (e.g., Strayer et al., 2013; Townsend \& Eideles, 2011; Townsend \& Wenger, 2004), but we focused on time production because it was validated as an efficient method which is not based on subjective evaluation (e.g., Zakay et al., 1999). Duration productions were longer under conditions of high physical load, which is predicted by an attentional-gate model (Zakay \& Block, 1997), among others.

Our finding that physical (psychomotor) and cognitive tasks reveal the same directional effects on duration estimation are intrinsic to much research on duration estimation. Since the earliest studies (e.g., MacDougall, 1913), researchers have attempted to evaluate the influence of various task demands. Unfortunately, these varieties of cognitive workload demand were rarely selected or organized in any coherent way, and such tasks were periodically interspersed with tasks like tapping. To some degree, all cognitive tasks combine some components of physical or psychomotor demand because participants must necessarily respond or act to withhold a response. Physical workload demands also contain necessary cognitive components. The empirical question is: what is the extent of interacting (moderating) effects, and what processes are involved?

Our present findings reveal that physical workload affects prospective timing in a similar way to cognitive workload tasks. However, this does not necessarily mean that the processing underlying the effects of physical and cognitive load are the same. It can be assumed that there are similarities but also dissimilarities between the two. Support for this comes from a study that we described earlier (Paas \& Adam, 1991). Another potential difference relates to the sense of agency, a term that refers to the experience of being in control of one's actions and their consequences. Demanet, Muhle-Karbe, Lynn, Blotenberg, and Brass (2013) found that the experience of effort directly contributes to the sense of agency. Rohde, Scheller, and Ernst (2014) found that temporal order between action and sensations is the dominant temporal cue for agency. In this respect there is a factor, unique to physical effort, that affects duration judgments.

The similarity between cognitive and physical load is that both of them require attentional resources, but the use of these resources might be task specific. Nevertheless, because prospective duration judgments rely on attentional resources, physical and cognitive workloads have similar effects on prospective duration judgment.
Weybrew (1963)

Warm et al. (1967)

Bortolussia et al. (1968)

Arlin (1989)

Vercruyssen et al. (1989)

Baldauf et al. (2009)

Buscà et al. (2011)
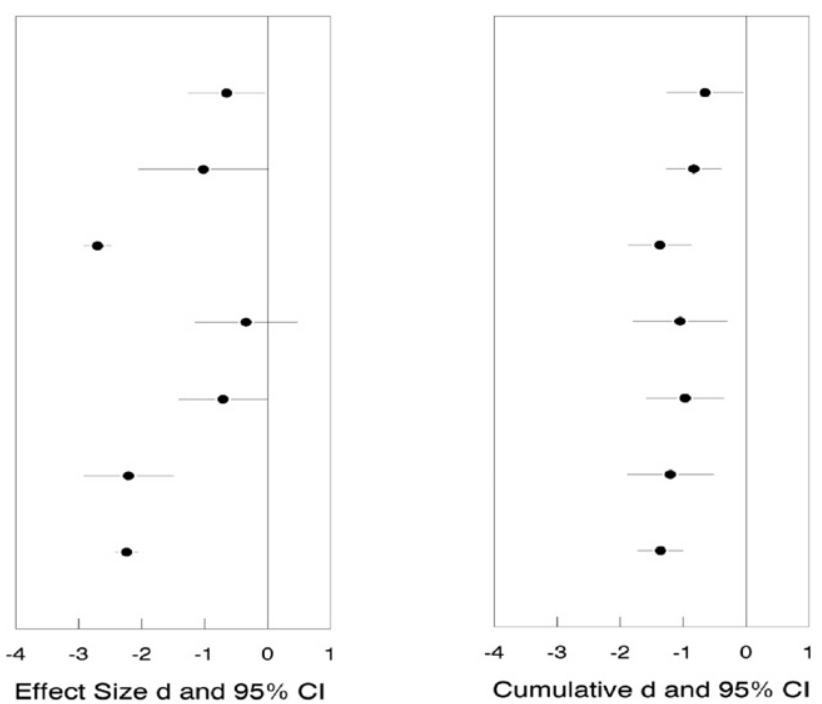

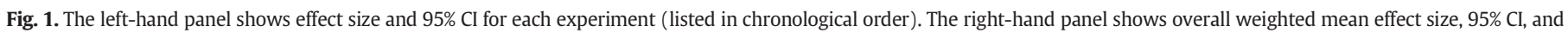
associated $p$ values (accumulated in chronological order). 
An illustration of the implication of the present findings can be related to sport psychology, a field in which the role of physical load is distinguished. In this domain, there are two competing models: one model (Scott, Scott, Bedic, \& Dawd, 1999) assumes that when physical effort is low, attention is allocated to the physical task; but when the effort is high attention is allocated to external factors. Another model (Tenenbaum \& Connolly, 2008) proposes the opposite: attention can be greater for timing than for external information-processing demands, but when the external task demands are high, subjects must allocate more attention to that task. Some empirical findings support Tenenbaum and Connolly's model, but these data are not yet conclusive. Our findings support Tenenbaum and Connolly's model: impairment of duration judgment is higher when physical effort is high than when it is low, in contradistinction to the predictions of Scott et al.'s model.

A question might be raised regarding extreme levels of physical effort, stress, and pain, in which verbal duration estimated lengthen (hence, productions shorten). This is the kind of phenomenon that occurs with earthquakes, in which duration are verbally overestimated. It was recently revealed in some initial eyewitnesses' verbal estimates of a stressful 6.0 magnitude earthquake near Napa, California, on August 24,2014 . Some residents reported a duration of about $25-30 \mathrm{~s}$, but later seismographic evidence revealed an actual duration of only $15 \mathrm{~s}$ (Buckhout, Fox, \& Rabinowitz, 1989). To address these issues, we suggest a model that is an elaboration of Tenenbaum and Connolly's (2008) model. When physical effort is low, attention is allocated to the primary information-processing task because few resources are needed to ensure appropriate performance. In such a case, duration judgments are only moderately affected. However, if primary task demands are high, attention required for prospective duration judgment is severely affected. What we add to the model is that if the primary task cannot benefit from additional attention because it is too difficult, stressful, or painful, resources are reallocated for timing. The main issue is therefore when the pain or other stresses will end. The meaning of this model is that up to a certain level of stress, pain, and difficulty, the regular rules of multitasking hold. Beyond a certain level of pain or other sources, most attentional resources are allocated for timing. This model can provide an explanation for the entire range of the phenomena.

In this meta-analysis, we were only able to assess the prospective paradigm, and thus we propose dual-task interference in attentional strategies as an explanation for the overall findings. However, we acknowledge the possibility of simple structural interference. In many dual-task performance situations, in which both physical and cognitive activities are engaged, decrements in cognitive performance might be erroneously attributed to simple physical interference. Thus, a body in motion tends to disrupt the otherwise facile process of information processing. For example, failures in concomitant memory performance may be attributed to the fact that a person cannot perceive the displayed memory set on a moving display, as opposed to a failure of the memory process per se. Indeed, in all studies of performance under stress, the elimination of such structural interference is a critical first consideration (Hancock \& Warm, 1989).

In the practical assessment of workload, not all methods are reliable and valid (e.g., Wierwille and Connor, 1983; Wierwille, Rahimi, \& Casali, 1985). Prospective temporal production is one of the methods that can eliminate or de-emphasize the role of structural competition (see also Townsend \& Eideles, 2011; Townsend \& Wenger, 2004). Thus, the source of such a large effect on time estimation is that directed psychomotor activity demands extensive attentional resources.

\section{Conclusion}

Our findings reveal that prospective timing is a useful, noninterruptive method of assessing physical workload demands. We also note that there is a considerable lack of experimental studies on effects of physical load on duration estimation. This is interesting in that there were not enough extant experiments to conduct moderator-variable analyses. In addition, no experiment used for the retrospective paradigm. Finally, researchers should investigate the combined effects of physical and cognitive workload, especially in paradigms that use time estimation and timing.

\section{References $^{1}$}

*Arlin, M. (1989). The effects of physical work, mental work, and quantity on children's time perception. Perception \& Psychophysics, 45, 209-214.

*Baldauf, D. Burgard, E. \& Wittmann, M. (2009). Time perception as a workload measure in simulated car driving. Applied Ergonomics, 40, 929-935.

Block, R. A., Hancock, P. A., \& Zakay, D. (2010). How cognitive load affects duration judgments: A meta-analytic review. Acta Psychologica, 134, 330-343.

Borenstein, M., Hedges, L. V., Higgins, J. P. T., \& Rothstein, H. R. (2011). Comprehensive meta-analysis (version 2.2.064) [computer software]. Englewood, NJ: Biostat.

*Bortolussi, M. R., Kantowitz, B. H., \& Hart, S. G. (1986). Measuring pilot workload in a motion base trainer: A comparison of four techniques. Applied Ergonomics, 17 278-283.

Buckhout, R., Fox, P., \& Rabinowitz, M. (1989). Estimating the duration of an earthquake: Some shaky field observations. Bulletin of the Psychonomic Society, 27, 375-378.

*Buscà, B., Moras, G., Seirulo-Lo, F., \& Cabot, J. (2011). Children's time production for concurrent nontemporal motor tasks. Perceptual and Motor Skills, 112 $151-160$.

Cohen, J. (1988). Statistical power analysis for the behavioral sciences (2nd ed.). Hillsdale, NJ: Erlbaum

Demanet, J., Muhle-Karbe, P. S., Lynn, M. T., Blotenberg, I., \& Brass, M. (2013). Cognition, $129,574-578$

Eccleston, C., \& Crombez, G. (1999). Pain demands attention: A cognitive-affective model of the interruptive function of pain. Psychological Bulletin, 125, 356-366.

Hancock, P. A., \& Block, R. A. (2012). The psychology of time: A view backward and forward. American Journal of Psychology, 125, 267-274.

Hancock, P. A., \& Manser, M. P. (1997). Time-to-contact: More than tau alone. Ecological Psychology, 9, 265-297.

Hancock, P. A., \& Warm, J. S. (1989). A dynamic model of stress and sustained attention. Human Factors, 31, 519-537.

Hancock, P. A., Arthur, E. J., Chrysler, S. T., \& Lee, J. (1994). The effects of sex, target duration, and illumination on the production of time intervals. Acta Psychologica 86, 57-67.

Hancock, P. A., Vercruyssen, M., \& Rodenburg, G. J. (1992). The effect of gender and time-of-day on time perception and mental workload. Current Psychology, 11, $203-225$

Hedges, L. V. (1981). Distribution theory for glass's estimator of effect size and related estimators. Journal of Educational Statistics, 6, 107-128.

Hedges, L. V., \& Olkin, I. (1985). Statistical methods for meta-analysis. Orlando, FL: Academic Press.

Horenstein, A. D., \& Rotter, G. S. (1969). Research methodology in temporal perception. Journal of Experimental Psychology, 79, 561-564.

MacDougall, R. (1913). Rhythm, time and number. American Journal of Psychology, 13, 88-97.

Paas, F. G., \& Adam, J. J. (1991). Human information processing during physical exercise Ergonomics, 34, 1385-1397.

Rohde, M., Scheller, M., \& Ernst, M. O. (2014). Effects can precede their cause in the sense of agency. Neuropsychologia, 65, 191-196.

Scott, L. M., Scott, D., Bedic, S. P., \& Dawd, J. (1999). The effect of associative and dissociative strategies on rowing ergometer performance. The Sport Psychologist, 13, 57-68.

Strayer, D. L., Cooper, J. M., Turril, J., Coleman, J., Ward, N. M., \& Biondi, F. (2013). Measuring cognitive distraction in the automobile. Washington, D.C.: Foundation for Traffic Safety (www.AAAFoundation.org).

Tenenbaum, G., \& Connolly, C. T. (2008). Attention allocation under varied workload and effort perception in rowers. Psychology of Sport and Exercise, 9, 704-717.

Townsend, J. T., \& Eideles, A. (2011). Workload capacity spaces: A unified methodology for response time measures of efficiency as workload is varied. Psychonomic Bulletin E' Review, 18, 659-681

Townsend, J. T., \& Wenger, M. J. (2004). A theory of interactive parallel processing: New capacity measures and predictions for a response time inequality series. Psychological Review, 111, 1003-1035.

*Vercruyssen, M., Hancock, P. A., \& Mihaly, T. (1989). Time estimation performance before, during, and following physical activity. Journal of Human Ergology, 18, 169-179.

*Warm, J. S., Smith, R. P., \& Caldwell, L. S. (1967). Effects of induced muscle tension on judgment of time. Perceptual and Motor Skills, 25, 153-160.

*Weybrew, B. B. (1963). Accuracy of time estimation and muscular tension. Perceptual and Motor Skills, 17, 118.

Wierwille, W. W., \& Connor, S. A. (1983). Evaluation of 20 workload measures using a psychomotor task in a moving-base aircraft simulator. Human Factors, 25, 1-16.

\footnotetext{
${ }^{1}$ References marked with an asterisk $\left({ }^{*}\right)$ indicate studies includable in the metaanalysis.
} 
Wierwille, W. W., Rahimi, M., \& Casali, J. G. (1985). Evaluation of 16 measures of mental workload using a simulated flight task emphasizing mediational activity. Human Factors, 27, 489-502.

Wittmann, M. (1999). Time perception and temporal processing levels of the brain. Chronobiology International, 16, 17-32.

Zakay, D., \& Block, R. A. (1997). Temporal cognition. Current Directions in Psychological Science, 6, 12-16.
Zakay, D., Bibi, A., \& Algom, D. (2014). Garner interference and temporal information processing. Acta Psychologica, 147, 143-146.

Zakay, D., Block, R. A., \& Tsal, Y. (1999). Prospective duration estimation and performance. In D. Gopher, \& A. Koriat (Eds.), Attention and performance XVII: Cognitive regulation of performance: Interaction of theory and application (pp. 557-580). Cambridge, MA: MIT Press. 